\title{
LOS SUSTANTIVOS MONOMORFÉMICOS ANIMADOS E INANIMADOS EN EL ZAPOTECO DE SAN PEDRO MIXTEPEC (MÉXICO) ${ }^{1}$
}

\author{
Pafnuncio Antonio Ramos \\ Universidad de Sonora (México) \\ paf217@hotmail.com
}

Recibido: 03/03/2019 - Aprobado: 25/06/2019

DOI: doi.org/10.17533/udea.lyl.n76a05

\begin{abstract}
Resumen: Los sustantivos monomorfémicos del zapoteco de San Pedro Mixtepec se clasifican en inanimados y animados. Estos a su vez se e subclasifican en nombres nativos y no nativos. Mientras que los inanimados nativos y no nativos se refieren a cosas, los animados nativos aluden a términos de parentesco y a nombres animales. Por su parte, los animados no nativos se usan como nombres propios y de animales. El corpus utilizado para el análisis es una base de datos de 35 horas de transcripciones, el cual fue requerido para observar los nominales en sus formas aisladas y en construcciones sintácticas.
\end{abstract}

Palabras clave: nombre; monomorfémicos; inanimado; animado; zapoteco.

\section{THE MONOMORPHEMIC INANIMATE AND ANIMATE NOUNS IN SAN PEDRO MIXTEPEC ZAPOTEC}

\begin{abstract}
The monomorphic substantives of the Zapotec of San Pedro Mixtepec are classified as inanimate and animated. These in turn are subclassified into native and non-native names. While native and non-native inanimates refer to things, native animates allude to terms of kinship and animal names. Non-native animates are used as proper names and animal names. The corpus used for the analysis is a database of 35 hours of transcriptions, which was required to observe the nominals in their isolated forms and in syntactic constructions.
\end{abstract}

Key words: noun; monomorphemic; inanimate; animate; Zapotec.

1. Agradecimientos a la Comisión Nacional de Ciencia y Tecnología por el soporte económico otorgado mediante la beca de Estancias Posdoctorales al Extranjero 2017-2018. A la Universidad de California, Santa Bárbara, por su aceptación como pasante de posdoctorado y a la Universidad de Sonora por la recepción como profesor invitado en el programa de Maestría en Lingüística. Sin el apoyo de estas instituciones no hubiera sido posible elaborar el presente artículo. Agradecimientos también a la Mtra. Denisse Martínez por revisar la redacción del mismo artículo. 


\section{Introducción}

$\mathrm{E}$

n el presente artículo se estudiarán los sustantivos monomorfémicos animados e inanimados del zapoteco de San Pedro Mixtepec (a partir de ahora ZSPM). Los sustantivos son términos que se emplean para nombrar entidades. Estos nominales corresponden a una clase de palabras gramaticalmente establecida, y pueden funcionar como núcleos de frases nominales (Genetti, 2016, p. 10). ${ }^{2}$ Semánticamente, los sustantivos pueden clasificarse de acuerdo a sus características de individuación, siendo la distinción entre animado e inanimado una de las más frecuentes (Hopper \& Thompson, 1980). En la gran mayoría de los casos, los sustantivos animados se refieren a entidades vivas, aunque también hay lenguas que consideran como entidades animadas a las estrellas, el sol, la luna, entre otros. En cambio, los sustantivos inanimados denotan entidades inertes (Marlett, 2012, p. 2).

En el ZSPM existen dos tipos de nombres monomorfémicos: los animados y los inanimados. Ambos tienen subcategorías que hacen referencia a nominales nativos y no nativos. La subcategoría del grupo animado - nativo y no nativo-, pueden contener elementos humanos y animales. Por su parte, la subcategoría del grupo inanimado, solamente contiene elementos inertes. Esto es similar a lo que reportó Operstain, en general, para las lenguas otomangues y en específico para las lenguas zapotecas (Operstain, 2003, p. 167).

El orden que se seguirá para la explicación del presente artículo es el siguiente: después de la introducción, se presentarán las generalidades del ZSPM (§2), la lengua zapoteca y la comunidad de estudio (§2.1) y las características gramaticales básicas del ZSPM (§2.2). Posteriormente, se mostrará el análisis de los nombres monomorfémicos sin morfología derivativa en (§3), los nombres monomorfémicos animados $(\S 3.1)$ y los nombres monomorfémicos inanimados $(\S 3.2)$. Finalmente, se expondrán las conclusiones de la investigación.

\section{Generalidades}

\subsection{La lengua zapoteca y la comunidad de estudio}

2. «The term noun refers to a grammatically defined word class, whose members can function as the heads of a noun phrases» (Traducción del autor). 
El zapoteco de San Pedro Mixtepec es una de las variedades dialectales del idioma zapoteco cisyautepequeño (Beam de Azcona, 2018; Campbell, 2017; Smith Stark, 2007). Este idioma, junto con el zapoteco de los valles centrales y el zapoteco del istmo conforman el grupo lingüístico conocido como zapoteco central. El zapoteco central, el miahuateco y el zapoteco del norte conforman el zapoteco medular, y agregando, a este último grupo, el solteco y el zapoteco occidental, se conforma la macrolengua zapoteca.

San Pedro Mixtepec es una comunidad rural ubicada en la Sierra Sur del estado de Oaxaca, aproximadamente a $180 \mathrm{~km}$ de distancia de la ciudad capital. De acuerdo con los resultados del Censo de Población, el número de habitantes de San Pedro Mixtepec es de 1077 personas (INEGI, 2015). Del total de la población la mayoría son hablantes de la lengua zapoteca.

\subsection{Características gramaticales básicas del zapoteco de San Pedro Mixtepec}

El sistema fonológico del ZSPM está compuesto de veinticuatro fonemas consonánticos y dos aproximantes (Ramos, 2007; 2015). De estos fonemas se derivan las grafías con las cuales se conforma el sistema ortográfico consonántico utilizado para la escritura práctica de la comunidad de estudio, y es el mismo sistema ortográfico que se emplea en los ejemplos que aparecerán en el artículo. ${ }^{3}$ Véase (Ramos, 2015):

$\begin{array}{llllllll} & \text { Oclusivas } & \text { Fricativas } & \text { Africadas } & \text { Nasales } & \text { Laterales } & \text { Vibrantes } & \text { Glides } \\ \text { Grafías } & \langle\pi, \tau, \kappa, \kappa \mathrm{w}\rangle & \langle\phi, \sigma, \mathrm{x}\rangle & \langle\mathrm{ts}, \mathrm{ch}\rangle & \langle\mathrm{m}, \mathrm{n}\rangle & <\mathrm{l}\rangle & <\mathrm{r}\rangle & ---{ }^{--} \\ \text {fortis } & & & & & & \end{array}$

3. La ortografía que se utiliza para presentar los datos de la lengua zapoteca corresponde al sistema establecido en la región Sierra Sur del estado de Oaxaca. Esta ortografía contiene algunas grafías derivadas de ciertos fonemas propiamente zapotecos y que no existen en el español. Para que los ejemplos sean legibles tanto para lectores lingüistas y no lingüistas presento las correspondencias fonológicas de estos grafemas con sus respectivos símbolos del Alfabeto fonético Internacional-IPA. La $\langle\mathrm{kw}\rangle$ ortográfica corresponde fonológicamente al fonema labiovelar fortis $/ \widehat{\mathrm{kw}} /$, la $\langle\mathrm{gw}\rangle$ ortográfica se corresponde con el fonema labiovelar lenis / $/ \mathrm{gw} /$, la $\langle$ ts $\rangle$ se corresponde con el fonema fricativo alveolar fortis $/ \widehat{t s} /$, la $\langle\mathrm{dz}\rangle$ se corresponde con el fonema fricativo alveolar lenis $/ \widehat{\mathrm{dz}} /$, la $\langle\mathrm{x}\rangle$ con el fonema fricativo posalveolar fortis $/ \mathrm{J} /$, la $\langle\mathrm{x}\rangle$ con el fonema fricativo alveolar lenis $/ 3 /$, la $<\mathrm{ch}>$ con el fonema africado posalveolar fortis $/ \mathrm{t} f /$, la $<\mathrm{ch}>$ con el fonema africado posalveolar lenis $/ \widehat{\mathrm{d}_{3}} /$. Las grafías que no se presentan aquí son las que se emplean en español. 
Grafías $\langle\beta, \delta, \gamma, \gamma w\rangle \quad\langle\zeta, \underline{\mathrm{x}}\rangle \quad\langle\mathrm{dz}, \underline{\mathrm{ch}}\rangle \quad\langle\underline{\mathrm{n}}\rangle$ $\underline{\mathrm{n}}>\quad \underline{\mathrm{l}}>$ $\langle r\rangle$ $\langle y, w>$ lenis

Tabla 1. Inventario de consonantes ortográficas del ZSPM

En lo que respecta al sistema vocálico, este se constituye de seis timbres vocálicos (a, e, $\Theta$, , , o, y u) y un contraste entre dos tipos de voz: la voz modal y la voz no modal, similar a lo que se reporta para el zapoteco de Juchitán y el zapoteco de San Pablo Güila (Herrera, 2000; Arellanes, 2009). El contraste entre estos dos tipos de voz posibilita la existencia de dos tipos de vocales fonológicas: modales y no modales, de las cuales se derivan los dos tipos de vocales ortográficas que aquí se emplean (Véase tabla 2): ${ }^{4}$

\begin{tabular}{|l|l|l|l|}
\hline Altura & Anterior & Media & Posterior \\
\hline Alta & $<\mathrm{i}, \mathrm{i}^{\prime}>$ & & $<\mathrm{u}, \mathrm{u}^{\prime}>$ \\
\hline Media & $<\mathrm{e}, \mathrm{e}^{\prime}>$ & & $<\mathrm{o}, \mathrm{o}^{\prime}>$ \\
\hline $\begin{array}{l}\text { Media } \\
\text { baja }\end{array}$ & $<$ ë, ë' $>$ & & \\
\hline Baja & & $<\mathrm{a}, \mathrm{a}^{\prime}>$ & \\
\hline
\end{tabular}

Tabla 2. Inventario de vocales ortográficas del ZSPM

El ZSPM es una lengua tonal con un inventario de cinco tonemas: el tono bajo, el alto, el bajo ascendente, el alto ascendente y el descendente. Los cuatro primeros son tonos léxicos y el último es producto de un proceso de sandhi tonal.

\begin{tabular}{|c|c|c|}
\hline & & Forma ortográfica \\
\hline \multirow[t]{2}{*}{ Tonos de nivel } & Tono bajo & $<>$ \\
\hline & Tono alto & $\langle>>$ \\
\hline \multirow[t]{2}{*}{ Tonos de contorno } & Tono bajo ascendente & $\langle\vee>$ \\
\hline & Tono alto ascendente & $\langle "\rangle$ \\
\hline
\end{tabular}

4. Las primeras cinco vocales son las mismas del español. Sin embargo, el zapoteco cuenta con una sexta vocal, que en este caso es la vocal media baja anterior <ë> y que fonológicamente se representa como / $\Theta /$. Las vocales modales no requieren de ninguna otra anotación adicional al de la grafía, mientras que las vocales no modales llevan un apostrofe, lo que indica la glotalización, la laringización o la rearticulación vocálica. Por ejemplo, la grafía $\langle\alpha \ni\rangle$

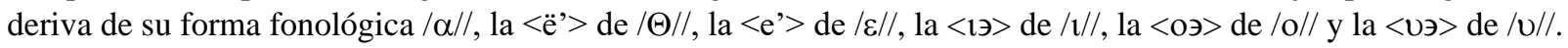




\begin{tabular}{|l|l|l|}
\hline & Tono descendente & $\left\langle{ }^{\wedge}\right\rangle$ \\
\hline
\end{tabular}

\section{Tabla 3. Inventario tonal del $\mathrm{ZSPM}^{5}$}

El ZSPM es una lengua de verbo inicial. En las construcciones intransitivas el orden es Verbo- Sujeto, como en (1a); en las construcciones monotransitivas, el orden es Verbo-AgentePaciente, como en (1b); en las construcciones ditransitivas de objeto oblicuo, el orden es VerboAgente-Tema-Recipiente, como en (1c); entre tanto, en las construcciones ditransitivas directas el orden de los constituyentes es flexible, como en (1d) y (1e). En los primeros tres tipos de construcciones presentados el orden es rígido, mientras que en las construcciones ditransitivas directas el orden es flexible.

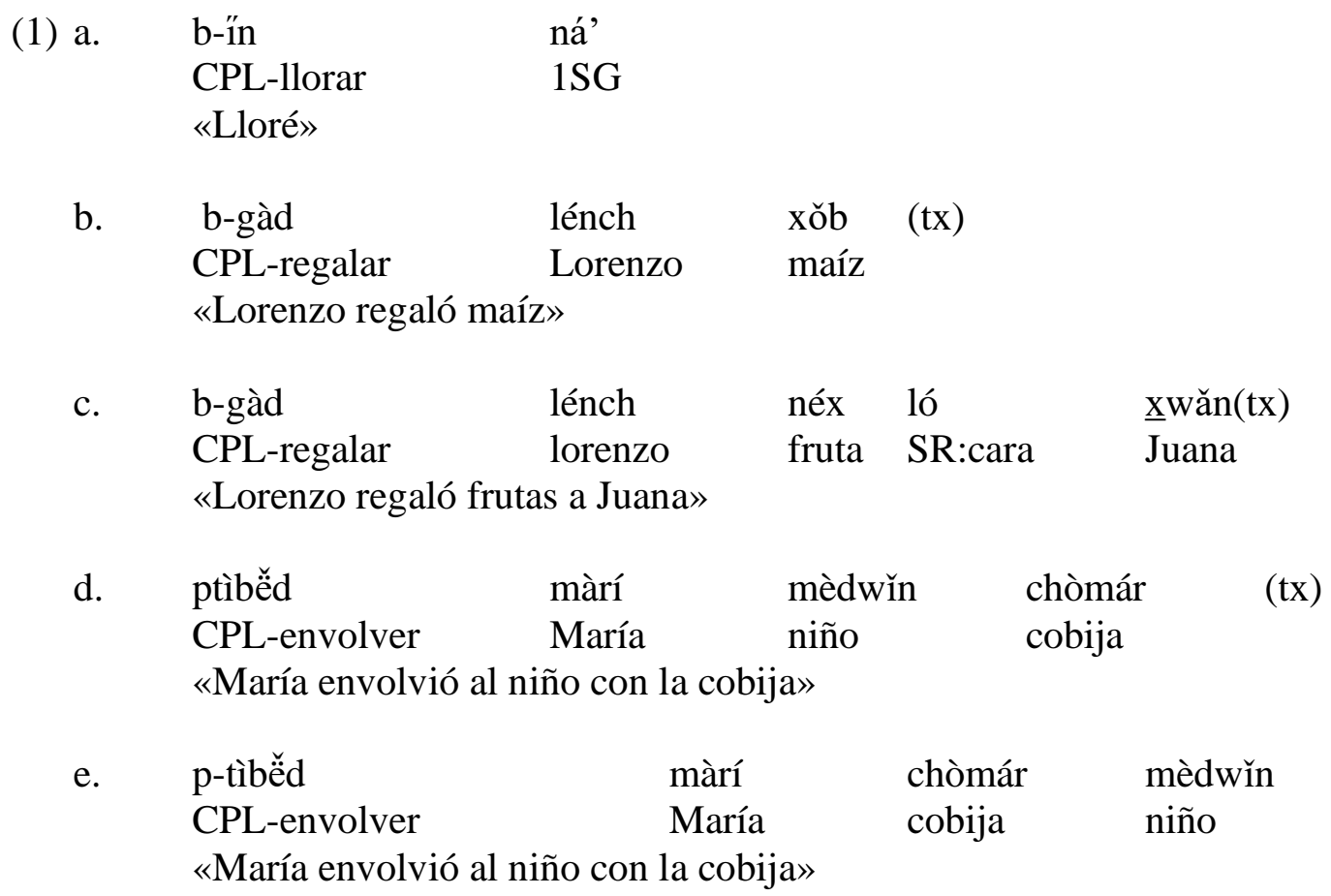

El sistema de alineamiento en construcciones monotransitivas es nominativo-acusativo, el cual se determina por el orden de los constituyentes. Por lo tanto, la posición nominativa se encuentra inmediatamente después del verbo, como en (2a), y posterior a él se encuentra la del acusativo, como en (2b):

5. Las abreviaturas que se usan en esta investigación son: $1 \mathrm{SG}=$ primera persona singular, 3DIST=tercera persona distal, $\mathrm{CPL}=$ completivo, ICPL=incompletivo, $\mathrm{POT}=$ potencial, $\mathrm{S}=$ sujeto, $\mathrm{SR}=$ Sustantivo relacional, $\mathrm{TX}=\mathrm{de}$ texto, $v=$ verbo. 
(2)
a. b-dín wël
bëd dzé nè
g-wèy=mé
lgř lă
$(\mathrm{tx})$
CPL-pegar Manuel
Pedro día que CPL-ir-3DIST
plaza Oaxaca

«Manuel le pegó a Pedro el día que fueron al tianguis en Oaxaca»

b. b-dín bếd wë́l dzé nè b-ròbnìs më̀dwǐn $(\mathrm{tx})$

CPL-pegar Pedro Manuel día que CPL-bautizar niños

«Pedro le pegó a Manuel el día que se bautizaron los niños»

Asimismo, al interior de las construcciones ditransitivas directas se observa un alineamiento neutro, ya que el objeto directo y el objeto indirecto pueden intercambiar su posición y la construcción sigue teniendo el mismo significado. (Véase 3a y 3b). Por otro lado, en construcciones ditransitivas con oblicuos el alineamiento es del tipo secundativo (Malchukov, 2010). El paciente y el tema no necesitan de ninguna marca para introducirse, en tanto que el recipiente se introduce con el sustantivo relacional lo «cara» (Véase 3c y 3d):

(3)
a. p-tìbë́d
màrí kùchí
chòmár
CPL-envolver
mari cuchillo
cobija
«María envolvió el cuchillo con la cobija»

b. p-tìbě̀d

màrí chòmár

kùchí

CPL-enrrollar Marí cobilla niño

«María envolvió el cuchillo con la cobija»

c. b-nëdz nà giét

CPL-dar $\quad$ 1SG tortilla

«Yo di tortilla»

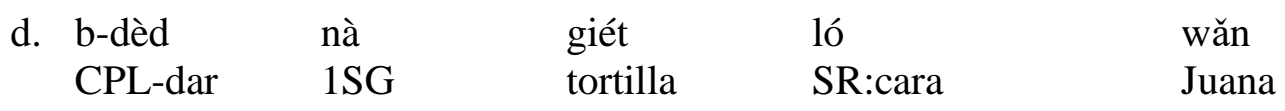

«Yo le di tortilla a Juana»

\section{Análisis de los nominales monomorfémicos}

Los nominales monomorfémicos del ZSPM se caracterizan por contar con dos mecanismos lingüísticos principales para clasificarse: i) la presencia o ausencia de un tono alto flotante y ii) la etimología de estos mismos elementos léxicos. El mecanismo de tono flotante organiza a estos 
nominales en dos principales clases: la clase nominal inanimada y la clase nominal animada. La primera clase (la nominal inanimada), se caracteriza por tener la presencia de un tono alto flotante, mientras que la segunda clase (nominal animada), se distingue por la ausencia del tono alto flotante. La clase de los inanimados, a diferencia de los animados, presenta tres procesos tonales: I) ascenso tonal del elemento léxico portador del tono alto flotante, II) bloqueo del ascenso tonal de la palabra anterior al elemento léxico portador del tono alto flotante, y III) la estrategia de tono flotante opcional.

Por otra parte, el mecanismo de etimología de los sustantivos monomorfémicos animados e inanimados establece una subclasificación que depende del origen de estos, organizándolos así en sustantivos nativos y en sustantivos no nativos. Los sustantivos inanimados nativos y no nativos se refieren a entidades inertes, cuyos únicos sustantivos con procesos fonológicos adicionales, como la elisión y fortición son los no nativos, mientras que los nativos no recurren a ningún otro proceso adicional. Entre tanto, los sustantivos animados recurren a la etimología de las palabras para subcategorizarse, como el caso de los sustantivos nativos animados, los cuales se subclasifican en humanos y animales. Mientras que los humanos se subcategorizan en términos de parentesco y nombres propios, los animales aluden únicamente a nombres de animales.

Un porcentaje importante de estos nominales son considerados primitivos léxicos, es decir, sustantivos nativos, mientras que los restantes son léxicos tomados como préstamos del español, los cuales se han adaptado a la estructura fonológica y silábica de la lengua zapoteca con el paso del tiempo.

\subsection{Nombres de entidades inanimadas}

Los nombres monomorfémicos inanimados no presentan ningún tipo de morfología, ya sea derivativa o flexiva. La raíz misma es la que le da el significado al nominal. Una característica principal que distingue a estos sustantivos de los animados es que presentan un sandhi tonal. El sandhi tonal es el cambio que ocurre en los sustantivos inanimados al participar en una construcción sintáctica ${ }^{6}$.

7. Para mayor detalle sobre la temática de sandhi tonal véase Ramos (2015), McIntosh (2015), Cruz \& Woodbury (2006a), Nelson (2004), Bickmore \& Broadwel (1998). 
Por ejemplo, una palabra de tono bajo en su forma léxica aislada puede cambiar a un tono alto o a un tono descendente al participar en una frase u oración. En (4a) por ejemplo, la palabra da $\Upsilon$

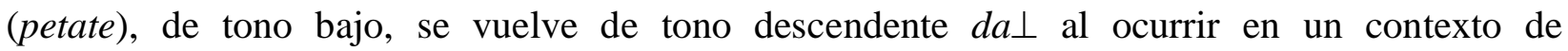
construcción sintáctica. Este proceso no ocurre cuando el sustantivo es animado, como me $\eta l$ (pescado), que también es de tono bajo, pero que conserva su tono aun participando en frases u oraciones, como en ¡Error! No se encuentra el origen de la referencia.:

(4)

a.

\begin{tabular}{|c|c|c|c|c|}
\hline r-lădz & ná & y-kă & nà & dâ \\
\hline ICPL-querer & $1 \mathrm{SG}$ & POT-comprar & $1 \mathrm{SG}$ & petate' \\
\hline \multicolumn{5}{|c|}{ «Quiero comprar petate» } \\
\hline r-lădz & ná & y-kă & ná & mèl \\
\hline ICPL-querer & $1 \mathrm{SG}$ & POT-comprar & $1 \mathrm{SG}$ & pescado \\
\hline
\end{tabular}

Asimismo, existen sustantivos inanimados que estando en esta misma posición sintáctica no cambian de tono, como es el caso de las palabras con tono bajo ascendente. Sin embargo, estas presentan un proceso diferente, el cual consiste en bloquear el ascenso tonal de la sílaba anterior, como en (5a). Dicho proceso no ocurre al tratarse de sustantivos animados de tono bajo ascendente, como en $(5 b)$ :

\begin{tabular}{|c|c|c|c|c|}
\hline r-lădz & ná & y-kă & nà & měx \\
\hline ICPL-querer & $1 \mathrm{SG}$ & POT-comprar & $1 \mathrm{SG}$ & mesa \\
\hline \multicolumn{5}{|c|}{ «Quiero comprar una mesa» } \\
\hline r-lădz & ná & y-kă & ná & mě \\
\hline ICPL-querer & $1 \mathrm{SG}$ & POT-comprar & $1 \mathrm{SG}$ & gorrión \\
\hline
\end{tabular}

Un tercer grupo de sustantivos inanimados son los de tono alto y alto ascendente. Estos manifiestan el sandhi tonal de manera opcional, lo que significa que pueden comportarse como los sustantivos de tono bajo, como en (4a), o como los sustantivos de tono bajo ascendente, como en $(5 a)$.

Asimismo, además de los sustantivos inanimados monomorfémicos nativos, también existe un grupo de sustantivos inanimados tomados como préstamos del español. Algunos de estos 
préstamos se han adaptado a la fonología y estructura silábica de la lengua que en la actualidad no parecen ser préstamos, en tanto que otros, al ser más recientes, son más identificables.

3.1.1. Nombres de entidades inanimadas monomorfémicas nativas

El grupo monomorfémico inanimado está conformado por sustantivos de los cuatro tonos léxicos, los cuales presentan una estructura silábica simple CV, CVC, CVVC, CVV, CCV, CCVC. En los ejemplos del (6a) al (6c), se muestran casos de tono bajo; del (6d) al (6f), los de tono bajo ascendente; los del (6g) al (6i), los de tono alto; y los del (6j) al (61), los de tono alto ascendente.

(6) Nombres monomorfémicos inanimados sin morfología derivativa
a. dà «petate»
b. gièts «metate»
c. kiè «piedra»
d. băy «rebozo»
e. měx
«mesa»
f. sǔ'd «enredo»
g. giéx «queso»
h. méx «jilote»
i. blág
j. bzië «hoja» «pozo»
k. mzië «flor de calabaza»
1. bnëb «fotografía»

3.1.2. Nombres de entidades inanimadas no nativas

Tal como se mencionó anteriormente, los nominales monomorfémicos que son empleados para nombrar cosas que no son propias de la cultura zapoteca, generalmente se toman prestados del español. La mayoría proviene de palabras monomorfémicas y bimorfémicas hispánicas. Tales palabras, al utilizarse en zapoteco, pasan por ciertos procesos fonológicos con el fin de adaptarse a la fonología de la lengua.

El primer tipo recurre a un proceso de elisión vocálica final. Estas palabras son las que en la lengua fuente son bisilábicas, y que al mudarse al zapoteco se vuelven monosilábicas. El proceso fonológico que ocurre en este tipo de sustantivos es una elisión vocálica, en el cual la vocal que 
se elide es siempre la de la sílaba ligera y la que se conserva es la de la sílaba prominente. Adicionalmente, se conserva el inicio de segunda sílaba. Este tipo de palabras, al adaptarse al zapoteco, se le asigna el tono alto, aunque la lengua fuente no sea tonal. (Véase los ejemplos en 7):

(7) Nombres bisilábicos a monosilábicos que sufren una elisión vocálica final
a. plát «plato»
b. lát «lata»
c. líkr «licra»
d. pèlót «pelota»
e. bàrrét «barreta»

El segundo subtipo de sustantivos no nativos es originalmente bisilábico, pero que al pasar a la lengua de destino se vuelven monosilábicos. El primer proceso al que recurren es a la elisión vocálica final de la sílaba ligera. El segundo proceso es una mutación consonántica por punto y modo de articulación, en el cual el fonema fricativo alveolar fortis /s/ se convierte en fonema fricativo pasalveolar lenis /Z/, que ortográficamente se representa / $\underline{x} /$. Estos nombres, al igual que los casos anteriores, pertenecen a los sustantivos inanimados. El tono que se le asigna a estos préstamos es el tono bajo ascendente, los cuales se pueden apreciar en los ejemplos de (8a) y (8b):

(8) Nombres bisilábicos con proceso de elisión vocálica final y mutación consonántica a. měx «mesa»

b. mǐx «misa»

Cabe resaltar el caso de la palabra nìx que se originó de anís. Esta misma, al pasar a la lengua de destino reduce una de sus sílabas. Para ello, recurre a una elisión vocálica inicial que corresponde a la sílaba ligera. Adicionalmente, se observa la mutación consonántica del fonema fricativo alveolar /s/, que se convierte en una fricativa postalveolar lenis /3/. El tono de esta palabra es bajo.

En el ZSPM los préstamos son muy irregulares. Por ello, el hecho de clasificarlos por tipos de procesos fonológicos es imposible. Cada préstamo tiene especificaciones particulares, los cuales, en muchos de los casos, no se comparten con otros tipos de préstamos. 
Un ejemplo de ello es el sustantivo giéx (queso, en español), el cual para adaptarse al zapoteco pasó por varios procesos fonológicos, primero, por una elisión de la vocal final /o/, después, por una mutación consonántica donde la grafía $<q>$ que fonológicamente se representa con el fonema velar fortis $/ \mathrm{k} /$ pasa a ser una consonante oclusiva velar lenis /g/, finalmente, la mutación de las vocales del núcleo /ue/ que cambian a /ie/. Otro caso es el sustantivo azúcar, que se vuelve sukr. En la adaptación de este préstamo se observa la elisión de los dos fonemas vocálicos /a/. Hasta el momento no se han podido establecer patrones de comportamiento en los préstamos monomorfémicos porque, como ya se explicó, cada caso tiene un comportamiento específico.

\subsection{Nombres monomorfémicos de entidades animadas}

El segundo grupo de sustantivos monomorfémicos del zapoteco de San Pedro Mixtepec son los sustantivos animados, los cuales no requieren de ningún tipo de morfología ni tampoco requieren de alguna especificación tonal para manifestarse. Estos nombres se dividen en tres subtipos: I) términos de parentesco, II) nombres personales y III) nombres de animales.

\subsubsection{Los términos de parentesco}

Los términos de parentesco hacen alusión a sustantivos para nombrar a personas con los mismos lazos consanguíneos o con lazos de tipo político y familiar (Jáuregui, 1980). Por la semántica que presentan, estos sustantivos pertenecen a los sustantivos del tipo inalienable. Dichos elementos se caracterizan por presentar una relación parental cercana directa con el poseedor. Por lo tanto, no pueden separarse de la entidad poseedora, además de que son intransferibles, porque no pueden concederse a una segunda o tercera persona. En construcciones posesivas la mayoría de estos nominales no requieren de una marca morfológica para indicar que están poseídos, ya que la posesión la marcan con la simple yuxtaposición de sus dos constituyentes (palabra poseída más expresión poseedora), como en (9a) y (9b): 
a.

bèts juán

Hermano juan

«El hermano de Juán»

b.

pxóz juán

padre Juan

«El papá de Juan»

Sin embargo, se cuenta con pocos términos de parentesco que usen la marca de posesión empleada para sustantivos alienables, como en (10a) y (10b). Estos casos, al ser un grupo de sólo cuatro sustantivos, se consideran excepciones del grupo de los inalienables.

a.

X-nià' juán

«La mamá de Juan»

b.

X-măl juán

«a comadre de Juan»

Los términos de parentesco que se presentan de (11a -11j) no requieren de morfología posesiva para indicar una relación de posesión. Mientras que los ejemplos de (11k -11n) usan el morfema posesivo de los sustantivos alienables. El comportamiento de estos términos de parentesco es impredecible ya que términos tan cercanos como nă 'mamá' usan el morfema de posesión como si fueran alienables y pxèy 'tío' o xùs 'tía' que es un poco más lejano tiene un comportamiento inalienable.

(11) Términos de parentesco
a. bèts
«hermano de varón»
b. bziàn
«hermano(a) sexo opuesto»
c. pxèy
«tío»
d. xùs
«tía»
e. dăd
«padre»
f. tsiě'l
«cónyuge»
g. pxóz
«papá»
h. bël
«hermana entre mujeres» 

i. xùz
«tía»
j. xěy
«tío»
k. nă
«madre»
1. miàg
«nieto»
m. măl
«comadre»
n. mbál
«compadre»

\subsubsection{Los nombres nativos para animales}

Existe también un pequeño número de nombres monomorfémicos asignados para nombrar a los animales. Estos nombres, al igual que los anteriores, sólo tienen la raíz nominal y no requieren de morfología adicional. Por tal razón, los hace diferentes a los nombres de los animales que tienen un morfema nasal derivativo o a los de clase que aparecen de manera obligatoria en sus inicios (Smith, 2007; Beam de Azcona, 2004). En la lista de dichos nominales se presentan ejemplos de tono bajo, como en (12a), de tono alto, como en (12b) y (12c) de tono bajo ascendente, como en los casos del (12d) al (12m). No se encontraron ejemplos de tono alto ascendente.

(12) Nombres para los animales
a. chù'r «chogón»
b. gwáy
«bestia»
c. kóg
«tortolita»
d. běnch
«becerro»
e. dăm
«búho»
f. dzǐng
«colibrí»
g. kwǐl
«pájaro azul»
h. lǐd
«gavilán»
i. rîl
«caballo»
j. tsĕww
«fiera»
k. rúx
«zopilote»
1. yěts
«canario»
m.x $x$ lŭts
«golondrinas»

\subsubsection{Los nombres propios en el ZSPM}


La mayoría de los nombres propios empleados en el ZSPM son de origen hispánico. Estos, al emplearse en zapoteco, pasan por ciertos procesos para adecuarse a la estructura fonológica o silábica de la lengua de destino.

Una generalización para este tipo de palabras es que conservan siempre la sílaba prominente, y en los casos en los que la misma no tiene coda recurren también a conservar la consonante inicial de la sílaba posterior. Las sílabas débiles y las vocales que quedan de las sílabas posteriores a la sílaba fuerte también son elididas. Los tipos silábicos que tienden a ocurrir en el idioma de estudio para este tipo de sustantivos son: inicio (simple), núcleo (simple o complejo) y coda (simple o compleja).

Los procesos fonológicos a los que se recurre con mayor frecuencia son la elisión, la epéntesis y las mutaciones consonánticas. El proceso de elisión es el que siempre ocurre cuando solamente se requiere de un solo proceso, mientras que la epéntesis y las mutaciones entran en juego al requerirse de más de un proceso fonológico. Los dos tipos de palabras que se analizan en este estudio son los que en la lengua fuente tienen dos o tres sílabas.

En (13a), (13b) y (13c) se observan nombres hispánicos de dos sílabas, los cuales al pasar a la lengua zapoteca se vuelven monosilábicos. La estrategia que utilizan es la elisión vocálica de la sílaba débil, y de esta manera se mantiene la sílaba con mayor prominencia. Dentro del mismo grupo existen palabras que inician con vocales. Tales palabras eliden la vocal inicial por corresponder a la sílaba débil y conservan la segunda sílaba intacta por ser la sílaba prominente, como en (13d). Hay también casos en los que se observan dos elisiones: la elisión de la vocal inicial y de la vocal final, como en (13e). Adicionalmente, existen casos en los que se recurre a una elisión silábica, como en (13f), y otros en los que ocurre una elisión silábica y una semiconsonantización vocálica de /u/ a /w/, como en (13g). Esto último se da para conservar un inicio consonántico, lo que equivale a cumplir con los principios del inicio silábico obligatorio. Finalmente, un caso donde se elide la vocal de la segunda silaba y se epentetiza una consonante palatalizada, como en (13h). Véase los ejemplos.

(13) Nombres propios con estructura bisilábica que cambian a monosilábicos
a. pédr
«Pedro»
b. rrós
«Rosa»
c. márt
«Marta» 

d. bél
«Abel»
e. dél
«Adela»
f. muěl
«Samuel»
g. wếl
«Manuel»
h. yăn
«Ana»

Un segundo tipo de nombres propios no nativos son los que en la lengua fuente están constituidos por tres sílabas y que al emplearse en zapoteco se reducen a una sola sílaba. Para estos casos, la única sílaba que se conserva es la de mayor prominencia. Cuando la sílaba prominente de un ítem léxico carece de coda silábica, la elisión de la sílaba ligera no es total, debido a que la lengua prefiere mantener el inicio de la sílaba ligera como coda de la sílaba que se mantiene. Por otra parte, en los ítems léxicos cuyas sílabas prominentes carecen de diptongo y de ataque silábico se les agrega una consonante palatal.

En (14a) aparece la elisión de la primera sílaba y la elisión de la vocal de la segunda sílaba, en la cual la única sílaba que se conserva es la fuerte. Para este mismo tipo de nombres se muestran algunos casos, como en (14b), en la que la sílaba seleccionada es lons. Las dos vocales, tanto la inicial — que corresponde a la primera sílaba—, como la vocal final — que pertenece a la tercera sílaba-, son elididas. Adicional a este proceso, ocurre una mutación consonántica del fonema fricativo alveolar fortis $/ s /$, que cambia a una consonante africada postalveolar fortis $/ \mathrm{t} f /$. Un ejemplo es (14c), en el que se observan dos procesos de elisión: uno en la primera sílaba y otro en la vocal final, la cual corresponde a la tercera sílaba ligera, por lo que queda únicamente cint al ser la sílaba fuerte.

Además de dichas elisiones ocurre también una mutación del fonema fricativo alveolar fortis /s/, que se vuelve una consonante africada posalveolar Lenis / $\widehat{d} /$. Un proceso adicional que se observa en los datos es sobre /t/, el cual, por medio de un proceso de lenición, cambia a /d/, como en (14d) y que, opcionalmente, se puede mantener como /t/, como en (14c). En (14e) se presenta otro ejemplo, en el cual la sílaba que se mantiene como prominente es la segunda, mientras que tanto la primera sílaba como el diptongo de la tercera son elididas. Adicionalmente, se tiene una mutación consonántica, en la que la fricativa alveolar fortis /s/ se vuelve una africada posalveolar fortis $\overparen{\mathrm{t}} \mathrm{f}$. Al tratarse del género femenino se recurre a una epéntesis de una nasal alveolar después del núcleo vocálico, como en (14f), pero cuando se refiere al género masculino no ocurre 
la epéntesis del elemento nasal. Esto no opera cuando se trata del género masculino, como en $(14 \mathrm{e}) \mathrm{y}$ en $(14 \mathrm{~g})$.

Un tipo más de nombres propios son los que mantienen la última sílaba, porque esta sílaba es la prominente. En los siguientes ejemplos la fricativa alveolar fortis /s/ también se vuelve africada posalveolar fortis /ch/ y se elide la primera vocal del diptongo. En (14i) se tiene un caso excepcional en el que no se respeta la restricción de las sílabas prominentes, porque seleccionan la primera sílaba y no la tercera.

(14) Nombres propios de tres sílabas
a. tín «ustina»
b. lǒnch
«Alonso»
c. chínt $\ll \operatorname{Jacinta}(\mathrm{a}) »$
d. chǐnd «Jacinto(a)»
e. méch «Nemecio»
f. měnch «Nemecia»
g. nánch «Venancio»
h. chőn «Asunción»
i. kónch «Concepción»

El último tipo de nombres propios que se presentarán son los que originalmente tienen cuatro sílabas. Para este tipo solamente se han encontrado dos ejemplos. En ambos se eliden las dos primeras sílabas y los elementos vocálicos finales. Mientras que en el caso de (15a) se conserva como dǐn, que significa Bernardino, el de (15b) se mantiene como fás, que significa Bonifacio.

(15) Nombres propios con tres sílabas
a. dǐn
«Bernardino»
b. fás
«Bonifacio»

\subsection{Nombres monomorfémicos no nativos para animales}

El último grupo de palabras monomorfémicas que se explicarán son los nombres de animales. El proceso que ocurre con estos nominales es la elisión vocálica de la última sílaba. Para las palabras de dos sílabas se elide la vocal de la sílaba débil y se conservan la sílaba fuerte y el inicio de la segunda sílaba, como en (16a), (16b), (16c) y (16d). Con respecto a las palabras de 
más de dos sílabas, el único proceso importante es la elisión de la vocal final, es decir, la vocal de la última sílaba.

(16) Nombres monomorfémicos para animales
a. ós
«oso»
b. pát
«pato»
c. kóbr
«cobra»
d. tígr
«tigre»
e. kàméy
«camello»
f. èlèfánt
«elefante»

Del mismo modo, se cuenta con algunos casos de nombres que no sufren elisiones vocálicas. Estas palabras conservan la mayoría de sus segmentos. El único proceso que presentan es un alargamiento vocálico de la última sílaba. Los ejemplos se presentarán del (17a) al (17g):

(17) Palabras con proceso de alargamiento vocálico
a. chùpàmië:1 «chupamiel»
b. pàbòrreá:1 «pavorreal»
c. leő:n «león»
d. jàbàlí: «jabalí»
e. chàká:1 «chacal»
f. jàguá:r «jaguar»
g. lòmbrí:z «lombriz»

\section{Conclusiones}

En el zapoteco de San Pedro Mixtepec los sustantivos monomorfémicos se clasifican en dos tipos: los inanimados y los animados. Los inanimados se distinguen por tener el tono alto flotante, el cual se manifiesta únicamente en un contexto sintáctico. Por otra parte, los sustantivos animados se distinguen por no poseer dicho tono alto flotante. El tono alto flotante tiene tres manifestaciones: la primera, se observa en palabras con tono bajo, que cambian a tono alto o a tono descendente en contextos sintácticos; la segunda, es un bloqueo del ascenso del tono flotante de la palabra precedente al léxico disparador del tono alto flotante, que son siempre las palabras de tono bajo ascendente; y la tercera, trata sobre los tonos flotantes opcionales, que ocurren en palabras de tono alto y alto ascendente. 
Por otro lado, los nombres monomorfémicos inanimados se clasifican en nombres para cosas inertes, patronímicos y nombres de cosas no nativas. Entre tanto, los nominales animados se clasifican en nombres para humanos y nombres para animales. Dentro del grupo de los humanos se tienen los nombres de parentesco, los cuales a su vez son nominales nativos. También se cuentan con los nombres propios, que al transitar de la lengua fuente a la lengua de destino obligatoriamente pasan por varios procesos fonológicos, con el fin de adaptarse a la estructura fonológica y silábica de la lengua. Finalmente, se tienen los nombres para los animales, que bien pueden ser nativos y no nativos.

\section{Referencias bibliográficas}

1. Arellanes, F. (2009). El sistema fonológico y las propiedades fonéticas del zapoteco de San Pablo Güilá: Descripción y análisis formal. (Tesis doctoral), El Colegio de México, Ciudad de México, México.

2. Beam de Azcona, R. (2004). A Coatlan-Loxicha Zapotec Grammar. USA. (Tesis de doctorado), Universidad de California, Berkeley.

3. Beam de Azcona, R. (2018). Diversificación y convergencia: una reconstrucción del zapoteco de la Sierra Sur y la filiación del cisyautepequeño. (Ponencia presentada en el Seminario de diacronía y sincronía en lenguas tipológicamente diversas). Universidad de Sonora, noviembre 12 y 13 .

4. Bickmore L. \& Georg Aaron, B. (1998). High Tone Docking in Sierra Juarez Zapotec. International Journal of American Linguistics, 64, 37-67.

5. Campbell Eric, W. (2017b). Otomanguean Historical Linguistics: Exploring the Subgroups. Language and Linguistics Compass, 11(7), 1-23.

6. Cruz E. y Anthony W. (2006). El Sandhi de Los Tonos en el Chatino de Quiahije. En Proceedings of the Conference on Indigenous Languages of Latin-America II, Universidad de Texas at Austin. Recuperado de https://www.ailla.utexas.org/site/cilla2_toc.html

7. Genetti, C. (2016). How Languages Work: An Introduction to Language and Linguistics. Cambridge: Cambridge University Press. 
8. Herrera, E. (2000). Amuzgo and Zapotec: Two More Cases of Laryngeally Complex Languages. Anthropological Linguistics, 42(4), 545-564. Indiana University

9. Jáuregui, J. (1980). Las relaciones de parentesco. Nueva Antropología, 5(18), 179-200.

10. Hopper, P. J. \& Thompson, S. A. (1980). Transitivity in Grammar and Discourse. Language, 251-299.

11. McIntosh Justin, D. (2015). Aspects of Phonology and Morphology of Teotepec Eastern Chatino, USA. (Tesis doctoral) University of Texas at Austin.

12. Nelson Julia, L. (2004). Tone and Glottalization on Nominals in San Juan Mixtepec Zapotec. (Tesis de maestría), University of Texas at Arlington.

13. Ramos, P. (2007). Las Propiedades fonológicas y morfofonológicos del Zapoteco de San Pedro Mixtepec. México (Tesis de maestría), Centro de Investigaciones y Estudios Superiores en Antropología Social-CIESAS, Ciudad de México, México.

14. Ramos, P. (2015). La fonología y morfología del zapoteco de San Pedro Mixtepec. México (Tesis doctoral), Centro de Investigaciones y Estudios Superiores en Antropología Social (CIESAS), Ciudad de México, México.

15. Smith, T. (2007). Algunas isoglosas zapotecas, en Memorias del III Coloquio Internacional de Lingüística Mauricio Swadesh, UNAM e INALI, México (pp. 69-133).

16. Marlett, S. (2012). La Animacidad. En S. Marlett (Ed.), Los Archivos Lingüísticos Me'phaa. Recuperado de http://www.mexico.sil.org/sites/mexico/files/mephaaanimacidad.pdf 